\title{
木材形成过程中次生壁沉积和细胞程序性死亡的 分子调控机制
}

\author{
李慧 ${ }^{1,2,3}$, 郭晓莕 ${ }^{1}$, 刘雅琳 $^{1}$, 陆海 $^{1,2,3^{*}}$ \\ 1. 北京林业大学生物科学与技术学院, 北京 100083 ; \\ 2. 北京林业大学, 林木育种国家工程实验室, 北京 100083; \\ 3. 北京林业大学林木分子设计育种高精尖创新中心, 北京 100083 \\ * 联系人, E-mail: luhai1974@bjfu.edu.cn \\ 收稿日期：2019-06-11; 接受日期：2019-11-22; 网络版发表日期：2020-02-13 \\ 中央高校基本科研业务费专项(批准号: 2019ZY30)资助
}

摘要 木材的形成经历了维管形成层细胞的增殖, 木质部细胞的分化和扩张, 次生细胞壁的沉积和细胞程序性死 亡 (programmed cell death, PCD)的过程. 近年来, 基于遗传学和组学分析, 人们已经从模式植物拟南芥和杨树中鉴 定出许多调控次生壁沉积的的关键转录因子和转录抑制因子. 这些转录因子调控层次分明, 共同构成次生壁沉积 的转录调控网络, 不仅在次生壁组分木质素、纤维素、木聚糖等物质的生物合成过程中起重要作用, 而且可激活 下游调控细胞程序性死亡相关的水解酶, 启动木质部细胞的程序化死亡过程. 对这些基因的生物学功能和调控网 络进行解析, 为阐明木材形成的分子生物学机制奠定了理论基础. 本文综述了木材形成过程中次生壁沉积的转录 调控网络和细胞程序性死亡相关的酶学机制及其最新研究进展.

关键词木材, 次生壁, 转录因子, 程序性死亡, 蛋白酶

木材是重要的可再生资源，不仅可用作生物质能 源, 而且可作为建筑和家具生产以及造纸业的原材料. 木材是木本植物维管形成层分化产生的次生木质部连 年沉积、附加的结果. 次生木质部的发育是一个连续 的发育过程，包括维管形成层细胞的增殖，木质部细 胞的分化和扩张，次生细胞壁的沉积并伴随着细胞程 序性死亡(programmed cell death, PCD). 最终成熟的 次生木质部包括木质部薄壁细胞、木质纤维、导管和 管状分子(tracheary elements, TE) ${ }^{[1,2]}$.

木材的形成是一个严格受基因编码调控的复杂的
动态生物学过程, 解析参与木材形成过程中的关键调 控因子以及作用机制, 为利用分子生物学手段进行改 良木材性状，提高木材的生长速率和提高木材产量意 义重大. 目前研究木材形成的模式系统，除了杨树等 木本模式植物以外，草本植物拟南芥和体外诱导管状 分子分化的百日草悬浮细胞也是理想的研究木材形成 和管状分子分化的模式系统 ${ }^{[3]}$. 本文对调控次生壁的 形成, 细胞程序性死亡过程中关键因子特别是转录因 子和重要酶的生物学功能、作用机理及其调控网络的 最新研究进展进行了综述.

引用格式: 李慧, 郭晓莣, 刘雅琳, 等. 木材形成过程中次生壁沉积和细胞程序性死亡的分子调控机制. 中国科学: 生命科学, 2020, 50: 123-135 Li H, Guo X R, Liu Y L, et al. The molecular mechanism in secondary wall deposition and programmed cell death of wood formation (in Chinese). Sci Sin Vitae, 2020, 50: 123-135, doi: 10.1360/SSV-2019-0133 


\section{1 次生细胞壁形成的转录调控机制}

次生壁主要由纤维素、半纤维素(木聚糖、木葡 聚糖、甘露聚糖等)、木质素以及少量的结构蛋白和 酶构成 ${ }^{[4]}$. 次生壁的形成发生在木质部细胞径向生长 结束之后，木质部细胞的细胞壁加厚过程涉及纤维 素、半纤维素、细胞壁蛋白和木质素的合成和沉积. 木质素主要沉积在木质部导管和纤维等厚壁组织中, 可增加细胞壁的机械强度和疏水性，并且在机械支 撑、水分运输和病害防御中起重要作用 ${ }^{[5]}$.

\section{$1.1 \quad$ NAC转录因子}

次生壁的沉积由次生壁成分合成基因和次生壁转 录因子共同调控 ${ }^{[6]}$. 其中, NAC(NAM/ATAF/CUC)和 MYB转录因子分别作为第一级和第二级主开关, 共同 激活一系列下游转录因子和次生壁物质合成基因 ${ }^{[7,8]}$. 人们在拟南芥中鉴定出一些与次生壁形成相关的含有 NAC结构域的转录因子，主要包括VASCULAR-RELATED NAC-DOMAIN(VND1,2,3,4,5,6,7), SECONDARY WALL-ASSOCIATED NAC DOMAIN(SND1, SND2), NAC SECONDARY WALL THICKENING PROMOTING FACTOR(NST1, NST2, NST3)(图1, 表 1)，过表达任何一个转录因子都能导致本来不含木质 素的细胞中出现木质素的异位沉积 ${ }^{[31]}$. 这些NAC转录 因子之间功能存在冗余，为了克服基因功能的穴余性， 人们一般通过将转录因子与EAR抑制元件进行融合构 建载体，来抑制该类转录因子共同调控的下游基因的 表达，获得显性抑制(dominant repression)的转基因植 株，从而研究基因被显性抑制后对植株表型的影 响 ${ }^{[16,32]}$. VND1-7与木质部导管分化过程相关, 过表达 $V N D 1-7$ 可以诱导拟南芥和杨树表皮和叶肉等细胞分 化形成导管, 出现次生壁的异位积累 ${ }^{[9,10]}$. 将强抑制元 件SRDX分别与VND6或VND7融合后转化拟南芥，获 得显性抑制基因表达的转基因拟南芥中导管形成受 阻, 根生长发育受抑制 ${ }^{[10]}$. SND1是激活次生壁物质合 成途径的主要转录开关, $S N D 1$ 在束间维管束纤维和木 质部纤维中特异表达，通过融合SRDX元件显性抑制 $S N D 1$ 基因表达可导致纤维细胞壁明显变薄; 过表达 $S N D 1$ 能激活次生壁合成基因的表达, 导致非木质化的 细胞出现次生壁的异位沉积, 从而产生叶片卷曲, 植株 矮化的表型 ${ }^{[16]}$. 另外, NST1 和NST3也是调节次生壁加
厚的重要转录因子，同时敲除SND1和NST3会阻碍次 生壁增厚和纤维细胞中木质素沉积, 植株呈现倒伏, 不能直立生长的表型 ${ }^{[13]}$.

通过对NAC转录因子所调控的下游靶基因的启 动子进行截短和电泳迁移率检测, 人们发现了一段 19 $b p$ 的不完美的回文保守序列：(T/A)NN(C/T)(T/C/G) $\operatorname{TNNNNNNNA}(\mathrm{A} / \mathrm{C}) \mathrm{GN}(\mathrm{A} / \mathrm{C} / \mathrm{T})(\mathrm{A} / \mathrm{T})$, 该序列被称为 SNBE元件(secondary wall NAC binding element), 该 元件是NAC转录因子结合所必需的, 突变SNBE元件 中任何一个核苷酸都会导致NAC转录因子与之结合 的能力丧失 ${ }^{[33]}$. 全基因组分析发现，除了 SND1和 VND7直接激活的下游MYB等转录因子以外，一些与 次生壁合成、细胞程序性死亡相关基因启动子也存在 多个SNBE元件. 另外有研究报道，一些受VND6直接 调控的靶基因启动子上含有一段核心序列 CTTNAAAGCNA, 该序列被称为TERE元件(TE-specific cis-element), 该元件在管状分子中特异表达 ${ }^{[11]}$. 靶 基因中存在相同的顺式作用元件说明了 $\mathrm{NAC}$ 转录因 子所调控的基因具有一定的共性，将顺式作用元件突 变之后, 转录因子与靶基因的结合会发生改变 ${ }^{[33]}$. 在 研究木质部发育相关的未知基因时，通过对未知基因 的顺式作用元件进行分析，可预测未知基因是否可能 被这些与次生壁形成相关的关键转录因子所调控, 从 而为下一步研究提供思路.

\subsection{MYB转录因子}

在植物基因组中, MYB 是一类植物特异的转录因 子, 其 $\mathrm{N}$ 端含有一段约 51 52个氨基酸组成的MYB结构 域, 根据MYB结构域的个数, 可分为R1-MYB, R2R3MYB, R1R2R3-MYB三个亚类, 植物中绝大多数MYB 转录因子属于R2R3-MYB类亚家族. 拟南芥中一些调 控次生壁合成的MYB转录因子, 作为NAC转录因子的 下游靶基因被鉴定出来. MYB46和MYB83不仅是SND1 的靶基因, 同时也是VND6和VND7的靶基因. SND1除 了直接激活MYB46, MYB83, SND3, MYB103和KNAT7 外，还调控许多其他转录因子的表达，包括SND2, MYB85, MYB52, MYB54, MYB69, MYB42, MYB43 和MYB2 $0^{[34]}$. MYB46和MYB83在调控次生壁形成方 面具有冗余性，而且拟南芥 $m y b 46 m y b 83$ 双突变体的 次生壁表现出比nst1 nst3更严重的缺陷. 在拟南芥中 过表达MYB46 和 $M Y B 83$ 可以激活纤维素、木聚糖和木 

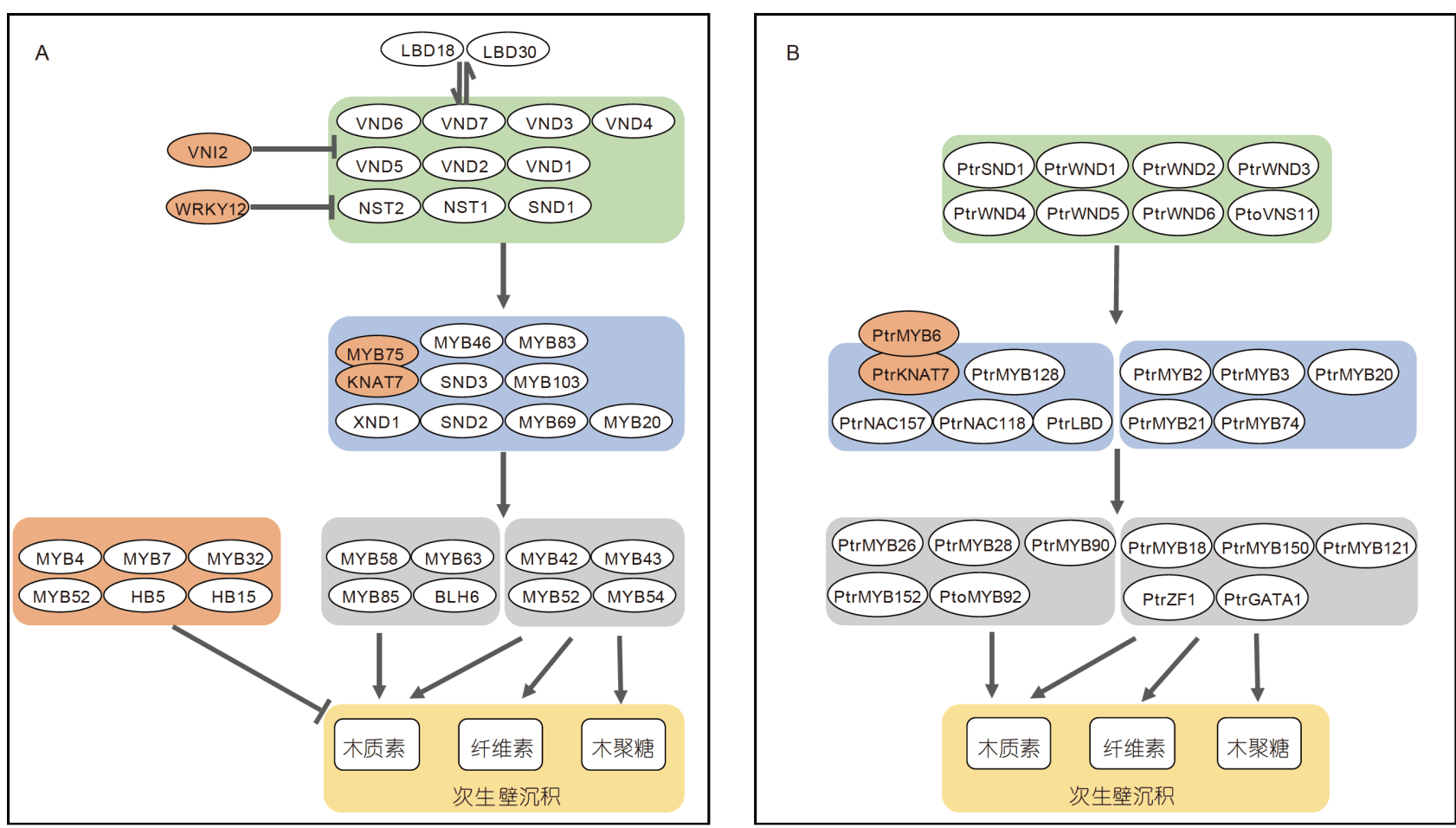

图 1 次生壁形成的转录调控网络, A: 拟南芥中次生壁形成的转录调控网络; B: 杨树中次生壁形成的转录调控网络. 绿色方 框: 一级转录调控; 蓝色方框: 二级转录调控; 灰色方框: 三级转录调控; 橙色方框和圆圈: 转录抑制因子; 白色圆圈: 转录因子; 实线表示直接调控; 虚线表示直接/间接调控未知; 箭头: 正调控, 平箭头: 负调控

Figure 1 The transcriptional regulatory network of secondary wall formation. A: The transcriptional regulatory network of secondary wall formation in Arabidopsis; B: The transcriptional regulatory network of secondary wall formation in Populus. Green block: The first layer of transcriptional regulatory network; blue block: the second layer of transcriptional regulatory network; grey block: the third layer of transcriptional regulatory network; orange block and circle: transcription repressor; white circle: transcription factor; solid line indicates directly regulation while dotted line indicates unknown directly or indirectly regulation; arrow: positive regulation; flat arrow: negative regulation

质素合成相关基因的表达，导致次生壁的异位沉积 ${ }^{[35]}$. 通过对MYB46所调控的下游基因启动子序列进行分 析发现，MYB46和MYB83通过结合一段7 bp 的保守 DNA序列SMRE元件(secondary wall MYB-responsive element)，来激活下游次生壁合成相关基因的表达. MYB46不仅能激活下游转录因子的表达，而且能激活 与木聚糖和木质素合成相关的基因表达 ${ }^{[36]}$.

木质素是植物次生细胞壁的主要成分，由芥子 醇、松柏醇、香豆醇三种单体聚合而成. 木质素单体 在细胞质中通过苯丙烷途径合成，然后转运到细胞壁 中聚合成木质素 ${ }^{[37]}$. 在苯丙烷代谢途径合成木质素的 过程中, R2R3-MYB转录因子发挥重要的调控作用 ${ }^{[38]}$. 研究发现, 木质素合成基因启动子中的AC元件是驱动 木质素合成的调节元件, 特定的MYB转录因子与 $\mathrm{AC}$ 元件结合，从而调控木质素合成基因的表达 ${ }^{[34]}$. 木质 素合成途径中的多个基因，如 $P A L, C 4 H, 4 C L, C 3 H$,
$C C O A O M T, \mathrm{CCR}$ 和 $C A D$ 的启动子区均含有一个或多 个AC元件, 包括AC-I(ACCTACC), AC-II(ACCAACC) 或AC-III(ACCTAAC) ${ }^{[39]}$. 在拟南芥中发现, 受NAC转 录因子调控的下游基因MYB58和MYB 63 与木质素的合 成有关, MYB58能直接与 $4 C L 1$ 的 $\mathrm{AC}$ 元件结合, 并激活 木质素合成基因的表达. 通过RNAi(RNA interference) 抑制MYB58和MYB 63的表达会导致拟南芥中木质素含 量下降, 次生壁变薄, 但并不影响纤维素和木聚糖的含 量; 而过表达会导致木质素的异位积累 ${ }^{[18]}$. 除NAC和 MYB两大家族的转录因子外, 还有很多其他的蛋白包 括Class III HOMEODOMIAN LEUCINE ZIPPER(HDZIP III) 转录因子和LATERAL ORGAN BOUNDARIES DOMAIN(LBD)家族蛋白, 也参与管状分子的 分化和次生壁的形成. 例如, 拟南芥中 $h b 5$ 发生突变后, 束间和束中纤维细胞中木质化程度增加; $h b 15$ 突变体 除了束间和束中纤维细胞中木质化程度增加，木质部 
表 1 拟南芥中调节次生壁形成的转录因子

Table 1 Transcription factors in regulating secondary wall formation in Arabidopsis

\begin{tabular}{|c|c|c|c|c|c|}
\hline \multicolumn{2}{|c|}{ 拟南芥 } & 转录因子 & 表达定位 & 功能 & 参考文献 \\
\hline \multirow{18}{*}{ 正调控 } & \multirow{10}{*}{ 一级主开关 } & VND1 & 维管组织 & 过表达: 次生壁异位沉积 & \multirow{5}{*}{ [9] } \\
\hline & & VND2 & 维管组织 & 过表达: 次生壁异位沉积 & \\
\hline & & VND3 & 维管组织 & $\begin{array}{c}\text { 过表达: 次生壁异位沉积; 抑制表达: } \\
\text { 减少导管细胞壁加厚 }\end{array}$ & \\
\hline & & VND4 & 维管组织 & 过表达: 次生壁异位沉积 & \\
\hline & & VND5 & 维管组织 & 过表达: 次生壁异位沉积 & \\
\hline & & VND6 & 后生木质部 & 参与PCD和次生壁形成调控 & {$[10,11]$} \\
\hline & & VND7 & 初生木质部 & 抑制表达: 抑制初生木质部的形成 & {$[10,12]$} \\
\hline & & NST1 & $\begin{array}{c}\text { 束间纤维和木质部纤维、 } \\
\text { 花药内皮层 }\end{array}$ & \multirow{2}{*}{$\begin{array}{c}\text { 调控茎纤维细胞和花药内层细胞次生壁加厚; } \\
\text { 功能存在冗余 }\end{array}$} & \\
\hline & & NST2 & $\begin{array}{c}\text { 束间纤维和木质部纤维、 } \\
\text { 花药内皮层 }\end{array}$ & & {$[7,13 \sim 15]$} \\
\hline & & NST3/SND1 & 束间纤维和木质部纤维 & $\begin{array}{c}\text { 过表达: 次生壁异位沉积; } \\
\text { 抑制表达: 减少纤维细胞壁加厚 }\end{array}$ & {$[13,16]$} \\
\hline & \multirow{2}{*}{ 二级主开关 } & MYB46 & 纤维和导管 & $\begin{array}{l}\text { 过表达: 次生壁异位沉积; } \\
\text { 抑制表达: 减少细胞壁加厚 }\end{array}$ & {$[15]$} \\
\hline & & MYB83 & 纤维和导管 & $\begin{array}{c}\text { 过表达: 次生壁异位沉积; } \\
\text { 抑制表达: 减少细胞壁加厚, 与MYB46功能攵余 }\end{array}$ & {$[17]$} \\
\hline & \multirow{3}{*}{$\begin{array}{l}\text { 特异调控木 } \\
\text { 质素合成 }\end{array}$} & MYB85 & 束间纤维和木质部 & $\begin{array}{c}\text { 过表达:在茎的表皮细胞、皮质细胞中产生木质素 } \\
\text { 异位沉积; } \\
\text { 显性抑制: 纤维细胞中细胞壁增厚显著降低 }\end{array}$ & {$[5]$} \\
\hline & & MYB58, MYB63 & 纤维和导管 & $\begin{array}{c}\text { 过表达特异激活木质素生物合成基因并同时伴有 } \\
\text { 木质素的异位沉积; } \\
\text { 显性抑制: 木质素含量降低, 次生壁增厚减弱 }\end{array}$ & [18] \\
\hline & & BLH6 & & 调控木质素形成 & [19] \\
\hline & \multirow{3}{*}{$\begin{array}{l}\text { 调控至少 } \\
\text { 一种组分 }\end{array}$} & SND2, SND3 & 纤维和木质部 & 过表达: 异位沉积; 抑制表达: 减少次生壁沉积 & {$[5]$} \\
\hline & & MYB103 & 纤维和木质部 & $\begin{array}{c}\text { 过表达: 纤维中细胞壁增厚; } \\
\text { 显性抑制: 纤维细胞中细胞壁增厚显著降低; } \\
\text { 调控S型木质素的合成 }\end{array}$ & {$[5,20]$} \\
\hline & & $\begin{array}{c}\text { MYB52, } \\
\text { MYB54, } \\
\text { MYB42, } \\
\text { MYB43, } \\
\text { MYB69, MYB20 }\end{array}$ & 纤维和木质部 & 抑制表达: 次生壁缺陷; 调控三种细胞壁组分 & {$[5,7]$} \\
\hline \multirow{8}{*}{ 负调控 } & & VNI2 & 木质部和韧皮部 & 抑制VND7的转录活性; 过表达导致导管缺陷 & [21] \\
\hline & & KNAT7 & 束间纤维和木质部 & $\begin{array}{c}\text { 突变体: 束间纤维细胞中次生壁厚度增加; } \\
\text { 过表达的表型相反 }\end{array}$ & {$[22,23]$} \\
\hline & & $\mathrm{XND} 1$ & 木质部 & 负调控PCD和次生壁合成 & {$[24,25]$} \\
\hline & & WRKY12 & & NST2和次生壁形成的转录抑制因子 & [26] \\
\hline & & MYB75 & & MYB75与KNAT7形成复合体抑制次生壁形成 & {$[23,27]$} \\
\hline & & OFP1, OFP4 & & 与KNAT7形成复合体抑制次生壁形成 & {$[22,28]$} \\
\hline & & $\begin{array}{l}\text { MYB4, MYB7, } \\
\text { MYB32 }\end{array}$ & & 木质素负调控因子 & [29] \\
\hline & & $\begin{array}{l}\text { MYB52, HB5, } \\
\text { HB15 }\end{array}$ & & $\begin{array}{c}\text { 木质素合成负调控因子, 突变体导致次生壁木质素 } \\
\text { 高度沉积 }\end{array}$ & {$[5,19]$} \\
\hline 其他 & & LBD18, LBD30 & & 正反馈调节VND7的表达 & {$[30]$} \\
\hline
\end{tabular}


细胞附近的薄壁细胞也发生木质素的异位沉积，而过 表达HB15 导致木质部区域减少 ${ }^{[19]}$. LBD 18/ASL20和 $L B D 30 / A S L 19$ 通过正反馈调节VND6与VND7参与管 状分子的分化, 其中, $L B D 30$ 为VND7的直接靶基因. 过 表达 $L B D 18$ 和 $L B D 30$ 的表型与过表达 $V N D 6$ 与 $V N D 7$ 的 表型一致, 均能诱导薄壁细胞产生管状分子 ${ }^{[30]}$.

\section{3 转录抑制因子}

另外，一些调节次生壁合成的负调控因子也被报 道, 包括XND1(XYLEM NAC DOMAIN 1), KNAT7 (KNOTTED1-LIKE HOMEODOMAIN PROTEIN7), VNI2(VND-INTERACTING2)和WRKY12等. 这些负 调控因子一方面通过直接与靶基因结合来调控次生壁 组成物的合成，另一方面通过与其他转录因子形成复 合物，间接调控下游基因的表达. 例如，KNAT7 是 $\mathrm{SND} 1$ 直接调控的靶基因 ${ }^{[5]}$, 丧失功能的knat 7 突变体 中, 束间纤维细胞壁厚度增加, 而过表达KNAT7的转 基因拟南芥和杨树均表现相反的表型 ${ }^{[22,40]}$. 在拟南芥 中过表达 $X N D 1$ 导致木质部管状分子的缺乏，胚轴木 质部区域缺乏，次生壁加厚 ${ }^{[24]}$. 而WRKY12可能位于 NAC转录的上游，体内外实验证明，WRKY12可直接 结合在 $N S T 2$ 基因的启动子区，抑制下游 $N S T 2$ 基因的表 达. WRKY12是调控髓细胞次生壁形成的转录抑制因 子，wrky12突变体髓细胞中的次生壁加厚，并且木质 素、木聚糖、纤维素增加, 茎中生物量积累增加 ${ }^{[26]}$.

有些转录抑制因子与其他转录因子形成复合体, 一起调控下游基因的表达. VND-INTERACTING2 (VNI2) 是一个NAC转录抑制因子, 可与 VND1-5, VND7结合，抑制木质部细胞的分化 ${ }^{[21]}$. MYB75是次 生壁形成的负调控因子, $m y b 75$ 突变体中纤维细胞次 生壁厚度增加, 同时木质素合成相关基因表达上调 ${ }^{[23]}$. 体内和体外实验均证明, KNAT7与MYB75形成蛋白复 合体, 共同调控次生壁的形成 ${ }^{[23,27]}$.

\section{4 杨树转录因子}

在杨树中, 存在类似的 NAC-MYB转录调控网 络 ${ }^{[41}$ (图2, 表2), 来自杨树的NAC转录因子(PtVNS/ PtrWND, WOOD-ASSOCIATED NAC DOMAIN)在功 能上已经被鉴定，12个PtVNS/PtrWND在木质部中表 达，过表达这些基因均能诱导杨树叶片和拟南芥幼苗 中次生壁增厚, 但是次生壁加厚的程度有所差异 ${ }^{[42]}$.
这些编码杨树 NAC转录因子(PtrWNDs)的基因, 不仅 在序列上与拟南芥 $S N D 1$ 存在相似性, 而且在功能上也 高度同源. 在拟南芥 $s n d 1$ nst 1 双突变体中表达这些 PtrWNDs, 均能使双突变体茎倒伏的表型恢复到正常 状态，且能使束间纤维细胞中次生壁的异常表型恢复 正常; 过表达P trWND2B和P trWND6B能够诱导一些次 生壁合成基因和相关转录因子的表达，同时也能诱导 纤维素、木聚糖和木质素的沉积 ${ }^{[43]}$. 通过研究杨树中 6 个与纤维素合成相关的MYB转录因子发现，过表达 PdMYB10，PdMYB199使木质纤维和束间纤维细胞的 细胞壁增厚, 而过表达PdMYB90, PdMYB167, PdMYB 92, PdMYB 125使纤维细胞的细胞壁变薄 ${ }^{[56]}$.

研究人员通过分析芯片表达数据，鉴定出多个在 木质部和维管组织发育过程中表达量较高的R2R3MYB 转录因子 ${ }^{[57,58]}$. 其中, 杨树中 P trMYB 3 和 PtrMYB20 是PtrWND2的靶基因, PtrMYB3 和PtrMYB20 是拟南芥 $M Y B 46$ 和 $M Y B 83$ 的同源基因, 在功能上也具 有同源性，在拟南芥 $m y b 46 m y b 83$ 双突变体中表达 PtrMYB3 或PtrMYB20可以弥补双突变体中导管细胞 壁的缺陷; 在拟南芥中过表达PtrMYB3或PtrMYB20能 促进纤维素、木聚糖和木质素的合成基因上调, 增加 纤维素、木聚糖和木质素的含量, 并且导致叶表皮细 胞中出现这些物质的异位沉积, 说明P trMYB3 和 PtrMYB20同时参与激活了这三种次生壁组成物的合 成途径 ${ }^{[35]}$. 通过对杨树PtrMYB2/3/20/21、桉树EgMYB2和松树PtMYB4所结合的启动子元件进行分析 发现，一段7 bp的保守序列 $\mathrm{ACC}(\mathrm{A} / \mathrm{T}) \mathrm{A}(\mathrm{A} / \mathrm{C})(\mathrm{T} / \mathrm{C})$, 与 拟南芥的SMRE元件类似, 这些转录因子通过与下游 靶基因的SMRE元件位点结合调控次生壁合成相关基 因的表达 ${ }^{[48]}$.

在杨树中，PtrMYB6被鉴定出是控制花青素合成 和次生壁加厚的负调控因子, 与拟南芥一样, PtrMYB6与KNAT7一起结合形成蛋白复合体，共同抑 制木质素和花青素合成基因的表达 ${ }^{[53]}$, 说明不同物种 的MYB转录因子在调控次生壁合成方面存在一定的 共性.

随着组学技术和生物信息学的发展, 将转录组测 序(RNA-seq)与染色质免疫共沉淀(ChIp-seq)测序结合, 极大地丰富了木材形成相关的转录调控网络, 一些新 的调控木质部发育相关的转录因子被报道, 而且体外 实验证实，这些转录因子与下游靶基因的DNA，以及 


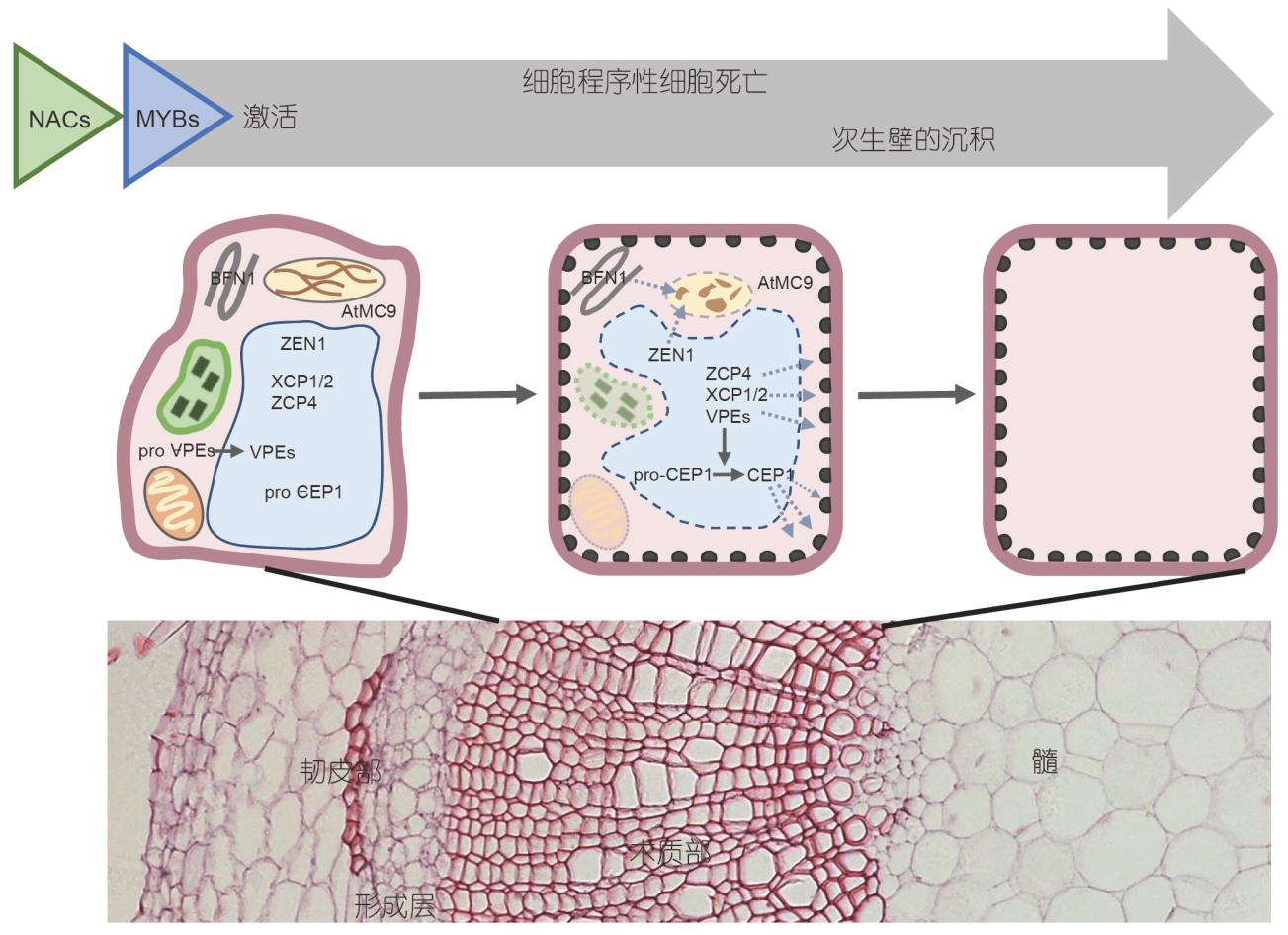

图 2 木质部细胞程序性死亡过程中的酶学调控机制

Figure 2 The enzymatic regulatory mechanism of PCD in xylem cells

转录因子之间的结合确实存在 ${ }^{[59]}$. 这些新发现的转录 因子在次生壁形成中的功能, 它们的表达水平发生改 变之后是否会影响木质部发育相关的表型改变, 以及 哪些次生壁组分会受到影响, 需要通过实验验证.

\section{2 细胞程序性死亡的酶学调控机制}

木质化结束后，管状分子和纤维细胞进入了细胞 程序性死亡阶段，管状分子的程序性死亡过程包括细 胞器的降解, 同时伴随着原生质体的降解和部分未木 质化次生壁的降解 ${ }^{[60]}$. 植物细胞的液泡裂解被认为在 管状分子的PCD过程中起关键作用, 其触发了木质部 细胞的程序性死亡. 当液泡裂解后, 液泡中的蛋白 酶、DNases和RNases等水解酶释放到原生质中, 继而 引发细胞内容物的完全降解 ${ }^{[60,61]}$.

\section{1 参与程序性死亡的核酸酶}

许多核酸酶参与了木质部细胞的程序性死亡过 程. 与植物 $\mathrm{PCD}$ 相关的核酸酶, 根据其所需离子的不 同, 分为 $\mathrm{Ca}^{2+} / \mathrm{Mg}^{2+}$ 依赖或 $\mathrm{Zn}^{2+}$ 依赖的的核酸酶, 以及
其他离子 $\left(\mathrm{Co}^{2+} / \mathrm{Mn}^{2+}\right)$ 依赖的核酸酶. 在百日草细胞诱 导细胞死亡的过程中发现，一个分子量大小为 $43 \mathrm{kD}$ 的 $\mathrm{Zn}^{2+}$ 依赖 $\mathrm{S} 1$ 类核酸酶ZINNIA ENDONUCLEASE1 (ZEN1), 能够特异性水解单链DNA. 该酶定位于液泡 中, 可能与细胞死亡有关 ${ }^{[62]}$. 随后研究证实, ZEN1是管 状分子降解细胞内容物的关键核酸酶, 在降解细胞核 DNA中起着重要作用, 反义抑制ZENI的表达抑制了 核DNA的降解，但是并不影响液泡裂解 ${ }^{[63]}$. 说明应该 还存在其他的酶类, 在管状分子的分化中起到降解液 泡和其他内容物的作用. 另外, 在拟南芥中还发现一 个分子量大小为 $38 \mathrm{kD}$ 的 $\mathrm{Zn}^{2+}$ 依赖核酸酶 $\mathrm{BFN} 1$, 同时 具有RNase和DNase活性, 既能降解RNA也能降解单 链DNA, 在衰老的花、叶和茎等多个组织中表达 ${ }^{[64,65]}$. 在叶片衰老过程中, 研究人员观察到BFN1从内质网转 运到细胞核, 最后定位于细胞核中, 介导细胞核中 DNA的降解, 从而参与器官衰老、木质部细胞成熟等 发育相关的多个PCD过程 ${ }^{[66]}$.

在毛白杨中, 人们发现一个在维管组织中特异表 达的 $\mathrm{Ca}^{2+}$ 依赖核酸酶PtCDD, 其能降解活体内的基因 组DNA, 过表达PtCDD可以促进导管的分化, 而抑制 
表 2 杨树中调节次生壁形成的转录因子

Table 2 Transcription factors in regulating secondary wall formation in Populus

\begin{tabular}{|c|c|c|c|c|c|}
\hline & 杨树 & 转录因子 & 表达定位 & 功能 & 参考文献 \\
\hline \multirow{14}{*}{ 正调控 } & \multirow{5}{*}{ 一级主开关 } & $\begin{array}{l}\text { PtVNS/ } \\
\text { PtrWNDs }\end{array}$ & 木质部 & 过表达: 次生壁异位沉积 & {$[42,43]$} \\
\hline & & $\begin{array}{l}\text { PtrWND2, } \\
\text { PtrWND6 }\end{array}$ & $\begin{array}{l}\text { 导管、纤维、韧皮部纤 } \\
\text { 维、射线薄壁细胞 }\end{array}$ & $\begin{array}{c}\text { 显性抑制: 次生壁厚度减少; 过表达: 次生壁异 } \\
\text { 位沉积 }\end{array}$ & {$[43,44]$} \\
\hline & & $\begin{array}{l}\text { PtrWND1, } \\
\text { PtrWND3, } \\
\text { PtrWND4, } \\
\text { PtrWND5 }\end{array}$ & $\begin{array}{c}\text { 导管、纤维、射线薄壁组 } \\
\text { 织 }\end{array}$ & 木材形成中的转录激活因子 & [43] \\
\hline & & PtoVNS11 & 维管组织 & 过表达: 木质素大量沉积和次生壁增厚 & {$[45]$} \\
\hline & & PtrSND1 & 木质部 & 木材形成中的转录激活因子 & {$[41,46,47]$} \\
\hline & \multirow{3}{*}{ 二级主开关 } & $\begin{array}{l}\text { PtrMYB3, } \\
\text { PtrMYB20 }\end{array}$ & $\begin{array}{l}\text { 导管、纤维、韧皮部纤 } \\
\text { 维、射线薄壁细胞 }\end{array}$ & $\begin{array}{c}\text { 过表达: 表皮细胞壁中木质素、纤维素、木聚 } \\
\text { 糖异位沉积 }\end{array}$ & {$[35]$} \\
\hline & & $\begin{array}{l}\text { PtrMYB2, } \\
\text { PtrMYB21 }\end{array}$ & 木质部导管、纤维 & $\begin{array}{l}\text { 过表达: 表皮细胞壁中木质素、纤维素、木聚 } \\
\text { 糖异位沉积; 显性抑制: 次生壁厚度减少 }\end{array}$ & {$[48]$} \\
\hline & & PtrMYB074/50 & 导管、木质部纤维 & PtrSND1的直接靶基因 & [41] \\
\hline & \multirow{6}{*}{ 其他 } & $\begin{array}{l}\text { PtSND2/ } \\
\text { PtrNAC154 }\end{array}$ & 木质部 & $\begin{array}{l}\text { 显性抑制; 次生壁厚度减少, 纤维素和木质素 } \\
\text { 含量下降; 过表达: 树皮增加, 次生木质部减少 }\end{array}$ & {$[49,50]$} \\
\hline & & PtrMYB28 & 次生木质部 & 激活木质素合成基因 & [34] \\
\hline & & PtrMYB152 & 木质部 & 过表达: 导管和纤维细胞壁厚度增加 & [51] \\
\hline & & PtoMYB92 & 木质部 & 过表达: 次生壁厚度增加, 木质素异位沉积 & [52] \\
\hline & & $\begin{array}{l}\text { PtrMYB26, } \\
\text { PtrMYB90, } \\
\text { PtrNAC156, } \\
\text { PtrNAC157 }\end{array}$ & 木质部 & $\begin{array}{c}\text { 被WND2 和WND6诱导, 至少调控一种次生壁 } \\
\text { 组分的基因表达 }\end{array}$ & {$[44]$} \\
\hline & & $\begin{array}{c}\text { PtrMYB18, } \\
\text { PtrMYB74, } \\
\text { PtrMYB75, } \\
\text { PtrMYB121, } \\
\text { PtrNAC150, } \\
\text { PtrZF1, } \\
\text { PtrGATA1 }\end{array}$ & 木质部 & $\begin{array}{c}\text { 被WND2和WND6诱导, 激活纤维素、木质 } \\
\text { 素、木聚糖基因表达 }\end{array}$ & {$[44]$} \\
\hline \multirow{5}{*}{ 负调控 } & & PtrKNAT7 & 维管组织 & $\begin{array}{c}\text { 过表达: 纤维细胞壁变薄; 突变体: 纤维细胞壁 } \\
\text { 增厚, 木质素增加 }\end{array}$ & {$[40]$} \\
\hline & & PtrMYB6 & 根、茎、叶 & $\begin{array}{c}\text { 过表达: 次生壁沉积减少, 次生壁合成基因的 } \\
\text { 表达受抑制 }\end{array}$ & {$[53]$} \\
\hline & & PopNAC122 & $\begin{array}{c}\text { 木质部、韧皮部、形成层 } \\
\text { 和树皮 }\end{array}$ & 过表达: 木质部发育受阻 & {$[50]$} \\
\hline & & PtrEPSP & 叶片原生质体细胞核中 & 过表达: 木质素异位沉积, 木质素含量增加 & [54] \\
\hline & & PttMYB021 & 木质部和韧皮部纤维 & 反义抑制: 植株变矮, 木质素含量增加 & {$[55]$} \\
\hline
\end{tabular}

$P t C D D$ 基因表达会延迟次生壁的沉积并影响次生木质 部的发育 ${ }^{[67]}$.

\section{2 参与程序性死亡的蛋白酶}

动物细胞 $\mathrm{PCD}$ 过程主要由半胱天冬氨酸蛋白酶类 (caspases)介导，该酶类通过水解天冬氨酸残基 $\mathrm{C}$ 末端 的肽键, 激活下游的caspases酶或分解细胞内相关的底 物蛋白, 导致细胞结构和代谢的改变, 最后引起细胞调 $亡^{[68]}$. 在植物细胞中, 并未发现该类酶的存在, 但在植 物中发现一些蛋白酶具有类似caspases的功能. 
对百日草悬浮细胞进行分析发现，管状分子的形 成需要半胱氨酸蛋白酶和丝氨酸蛋白酶的参与 ${ }^{[69,70]}$. 最早的药理学研究发现, 在百日草悬浮细胞培养体系 中添加蛋白酶抑制剂能够抑制管状分子内容物降解和 管状分子的分化 ${ }^{[71]}$. 在诱导管状分子分化的悬浮细胞 中不仅检测到蛋白水解酶的活性 ${ }^{[72]}$, 而且分离得到一 个编码 $20 \mathrm{kD}$ 大小的半胱氨酸蛋白酶基因 $p 48 h-17$, 该 基因与管状分子分化有关 ${ }^{[70]}$. 在百日草管状分子分化 过程中发现, 半胱氨酸蛋白酶ZCP4在导管分化早期表 达, 且ZCP4中 $11 \mathrm{bp}$ 的核酸序列(CTTGAAAGCAA) 是 管状分子特异表达所必需的元件 ${ }^{[59,73]}$. 随后，越来越 多的研究发现, 蛋白酶在木质部细胞的PCD中发挥重 要作用. 在杨树中发现一个具有caspase-3活性的20S蛋 白酶参与木质部发育，利用蛋白酶抑制剂可以抑制管 状分子的PCD过程 ${ }^{[74]}$. 两个在拟南芥木质部特异表达 的木瓜类半胱氨酸蛋白酶XYLEM CYSTEINE PROTEASE1(XCP1)和XCP2，与百日草ZCP4同源，它们在 管状分子中特异表达, 定位于液泡中, 可能作为自噬作 用的效应器参与导管分化 ${ }^{[75]} . x c p 1 x c p 2$ 双突变体的管 状分子中内容物降解不完全 ${ }^{[76]}$. 拟南芥AtMC9也是一 个在木质部中特异表达的金属蛋白酶, 参与调节导管 分子的自噬. 丧失功能的 $a t m c 9$ 突变体中表现出导管分 子自噬缺陷 ${ }^{[77]}$.

具有 caspase-1活性的液泡加工酶(vacuolar processing enzymes, VPEs)属于半胱氨酸蛋白酶legumain家 族，该酶类以无活性的蛋白前体形式在细胞质中合成， 进入液泡后, 在酸性环境下通过自我催化的方式转变 为成熟形式, 形成具有功能活性的酶 ${ }^{[78]}$. 当 $\mathrm{VPE}$ 被沉 默后，病毒诱导产生的PCD过程中液泡的裂解受到抑 制 ${ }^{[79]}$. 有报道指出VPE在杨树茎的木质部纤维中表 达 $^{[00,81]}$, 本团队 ${ }^{[82]}$ 研究发现, 拟南芥 $\gamma \mathrm{VPE}$ 在纤维细胞 的PCD中起重要作用, $\gamma v p e$ 突变体中纤维细胞内容物 降解延迟, 次生壁增厚且木质素含量增加. 另外, 本团 $队^{[83]}$ 研究发现, 木瓜蛋白酶(papain)家族的半胱氨酸蛋 白酶CEP1不仅在花药中表达, 参与调控线毡层的 $\mathrm{PCD}$ 过程; 也在茎的木质部细胞中特异表达, 参与调控导管 分子和纤维细胞的PCD过程. 当 cep 1 突变后, 拟南芥导 管分子和纤维细胞PCD过程受阻, 纤维细胞的次生壁 增厚 ${ }^{[84]} \cdot \gamma \mathrm{VPE}$ 与 CEP1均存在于木质部细胞中, 且在功 能上类似, 因此, 本团队猜测蛋白酶之间是否发生了级 联催化激活的过程, 从而导致细胞程序性死亡. 体外激
活实验证明, $\gamma \mathrm{VPE}$ 成熟酶能够激活CEP1成熟，在拟南 芥野生型植株中, 随着茎的发育成熟, CEP1能够完全 转变成成熟酶的形式, 而在 $\gamma v p e$ 突变体的成熟茎中, 还 有部分CEP1以酶原的形式存在 ${ }^{[82]}$. 说明先成熟的蛋白 酶VPE能够激活其他蛋白酶, 从而引发级联反应, 导致 液泡膜裂解, 进一步触发 PCD进程. 是否有其他蛋白酶 参与木质部细胞的程序性死亡过程? 这些酶之间是否 存在级联激活的关系, 哪些酶处于级联激活的上游, 哪 些酶处于下游? 随着越来越多相关研究的开展, 蛋白 酶之间的调控网络会逐渐被揭示.

次生壁形成与木质部细胞PCD是两个相互独立, 又相互依赖、紧密偶联的过程, 次生壁的形成和细胞 程序化死亡可能通过一个共同的信号级联途径调控, 但在某一点的调控上出现了差别. 调控次生壁形成的 关键转录因子的表达发生改变, 次生壁的形成受影响 的同时, PCD过程也会受影响, 在拟南芥中过表达 $X N D 1$ 不仅抑制次生壁的形成，也会抑制木质部管状 分子 $\mathrm{PCD}$ 过程 ${ }^{[24]}$. VND6 和VND7转录激活后, 不仅能 导致细胞壁形成相关基因的表达量上调, 也能导致细 胞死亡相关基因, 如 $X C P 1, X C P 2$ 和 $A t M C 9$ 的表达量上 调 ${ }^{[11]}$. $X C P 1$ 的启动子上含有 NAC转录因子结合的关 键元件SNBE序列, 能够与VND6, VND7和SND1结合, 说明 $\mathrm{NAC}$ 转录因子可能通过直接调控 $\mathrm{PCD}$ 相关的关 键酶基因的表达来调控细胞的死亡过程 ${ }^{[33]}$. 同样, 当 调控PCD过程的酶发生改变, 次生壁的沉积过程也会 受到影响. 例如, 调控PCD过程的半胱氨酸蛋白酶 $\mathrm{CEP} 1$ 发生突变后, 不仅纤维细胞和管状分子的细胞内 容物降解延迟，而且与细胞壁合成相关的基因表达上 调, 细胞壁的厚度增加 ${ }^{[84]}$. 说明次生壁形成与木质部 细胞PCD过程是相互偶联的过程, 与木质部发育相关 的关键转录因子除了通过与相关的蛋白酶基因的启动 子结合, 直接调控木质部细胞的PCD过程. 其是否也可 能通过影响蛋白之间的互作效率, 进而间接影响木质 部相关蛋白酶或合成酶的活性，这一过程值得进一步 探究.

\section{3 总结与展望}

植物木材的形成是复杂的生物途径, 众多转录因 子、酶、调控基因参与其中, 转录因子分级依次直接 或间接调控木材的形成, 且互相关联发挥促进或抑制 
作用. 有的直接调控次生壁形成和细胞程序性死亡过 程，有的间接调控这些过程，这些基因、酶、转录因 子之间形成了极为庞大的调控网络.

近年来, 随着分子生物学研究技术的不断发展, 木 材形成的分子调控网络不断被更新, 新的调控因子不断
被解析, 本文主要对调控木材形成的转录和酶学调控网 络进行了综述, 阐明了木材形成过程中次生壁形成和细 胞程序性死亡过程中的分子调控网络. 这些理论研究成 果将为分子水平上控制木材形成过程, 改良木材性状并 提高木本植物的经济价值提供重要的理论指导.

\section{参考文献}

1 Bollhöner B, Prestele J, Tuominen H. Xylem cell death: Emerging understanding of regulation and function. J Exp Bot, 2012, 63: 1081-1094

2 Turner S, Gallois P, Brown D. Tracheary element differentiation. Annu Rev Plant Biol, 2007, 58: 407-433

3 Nieminen K M, Kauppinen L, Helariutta Y. A weed for wood? Arabidopsis as a genetic model for xylem development. Plant Physiol, 2004, 135: 653-659

4 He X Q, Cui K M. Progress in study of secondary wall formation in plants (in Chinese). Chin Bull Bot, 2002, 19: 513-522 [贺新强, 崔克明. 植 物细胞次生壁形成的研究进展. 植物学通报, 2002, 19: 513-522]

5 Zhong R, Lee C, Zhou J, et al. A battery of transcription factors involved in the regulation of secondary cell wall biosynthesis in Arabidopsis. Plant Cell, 2008, 20: 2763-2782

6 Taylor-Teeples M, Lin L, de Lucas M, et al. An Arabidopsis gene regulatory network for secondary cell wall synthesis. Nature, 2015, 517: 571575

7 Zhong R, Ye Z H. Secondary cell walls: Biosynthesis, patterned deposition and transcriptional regulation. Plant Cell Physiol, 2014, 56: 195-214

8 Nakano Y, Yamaguchi M, Endo H, et al. NAC-MYB-based transcriptional regulation of secondary cell wall biosynthesis in land plants. Front Plant Sci, 2015, 6: 288

9 Zhou J, Zhong R, Ye Z H. Arabidopsis NAC domain proteins, VND1 to VND5, are transcriptional regulators of secondary wall biosynthesis in vessels. PLoS ONE, 2014, 9: e105726

10 Kubo M, Udagawa M, Nishikubo N, et al. Transcription switches for protoxylem and metaxylem vessel formation. Genes Dev, 2005, 19: 18551860

11 Ohashi-Ito K, Oda Y, Fukuda H. Arabidopsis VASCULAR-RELATED NAC-DOMAIN6 directly regulates the genes that govern programmed cell death and secondary wall formation during xylem differentiation. Plant Cell, 2010, 22: 3461-3473

12 Yamaguchi M, Kubo M, Fukuda H, et al. Vascular-related NAC-DOMAIN7 is involved in the differentiation of all types of xylem vessels in Arabidopsis roots and shoots. Plant J, 2008, 55: 652-664

13 Mitsuda N, Iwase A, Yamamoto H, et al. NAC transcription factors, NST1 and NST3, are key regulators of the formation of secondary walls in woody tissues of Arabidopsis. Plant Cell, 2007, 19: 270-280

14 Mitsuda N, Seki M, Shinozaki K, et al. The NAC transcription factors NST1 and NST2 of Arabidopsis regulate secondary wall thickenings and are required for anther dehiscence. Plant Cell, 2005, 17: 2993-3006

15 Zhong R, Richardson E A, Ye Z H. The MYB46 transcription factor is a direct target of SND1 and regulates secondary wall biosynthesis in Arabidopsis. Plant Cell, 2007, 19: 2776-2792

16 Zhong R, Demura T, Ye Z H. SND1, a NAC domain transcription factor, is a key regulator of secondary wall synthesis in fibers of Arabidopsis. Plant Cell, 2006, 18: 3158-3170

17 McCarthy R L, Zhong R, Ye Z H. MYB83 is a direct target of SND1 and acts redundantly with MYB46 in the regulation of secondary cell wall biosynthesis in Arabidopsis. Plant Cell Physiol, 2009, 50: 1950-1964

18 Zhou J, Lee C, Zhong R, et al. MYB58 and MYB63 are transcriptional activators of the lignin biosynthetic pathway during secondary cell wall formation in Arabidopsis. Plant Cell, 2009, 21: 248-266

19 Cassan-Wang H, Goué N, Saidi M N, et al. Identification of novel transcription factors regulating secondary cell wall formation in Arabidopsis. Front Plant Sci, 2013, 4: 189

20 Öhman D, Demedts B, Kumar M, et al. MYB103 is required for FERULATE-5-HYDROXYLASE expression and syringyl lignin biosynthesis in Arabidopsis stems. Plant J, 2013, 73: 63-76 
21 Yamaguchi M, Ohtani M, Mitsuda N, et al. VND-INTERACTING2, a NAC domain transcription factor, negatively regulates xylem vessel formation in Arabidopsis. Plant Cell, 2010, 22: 1249-1263

22 Li E, Wang S, Liu Y, et al. OVATE FAMILY PROTEIN4 (OFP4) interaction with KNAT7 regulates secondary cell wall formation in Arabidopsis thaliana. Plant J, 2011, 67: 328-341

23 Bhargava A, Mansfield S D, Hall H C, et al. MYB75 functions in regulation of secondary cell wall formation in the Arabidopsis inflorescence stem. Plant Physiol, 2010, 154: 1428-1438

24 Zhao C, Avci U, Grant E H, et al. XND1, a member of the NAC domain family in Arabidopsis thaliana, negatively regulates lignocellulose synthesis and programmed cell death in xylem. Plant J, 2008, 53: 425-436

25 Yamaguchi M, Demura T. Transcriptional regulation of secondary wall formation controlled by NAC domain proteins. Plant Biotech, 2010, 27: 237-242

26 Wang H, Avci U, Nakashima J, et al. Mutation of WRKY transcription factors initiates pith secondary wall formation and increases stem biomass in dicotyledonous plants. Proc Natl Acad Sci USA, 2010, 107: 22338-22343

27 Bhargava A, Ahad A, Wang S, et al. The interacting MYB75 and KNAT7 transcription factors modulate secondary cell wall deposition both in stems and seed coat in Arabidopsis. Planta, 2013, 237: 1199-1211

28 Liu Y, Douglas C J. A role for OVATE FAMILY PROTEIN1 (OFP1) and OFP4 in a BLH6-KNAT7 multi-protein complex regulating secondary cell wall formation in Arabidopsis thaliana. Plant Signal Behav, 2015, 10

29 Preston J, Wheeler J, Heazlewood J, et al. AtMYB32 is required for normal pollen development in Arabidopsis thaliana. Plant J, 2004, 40: 979995

30 Soyano T, Thitamadee S, Machida Y, et al. ASYMMETRIC LEAVES2-LIKE19/LATERAL ORGAN BOUNDARIES DOMAIN30 and ASL20/ LBD18 regulate tracheary element differentiation in Arabidopsis. Plant Cell, 2008, 20: 3359-3373

31 Grima-Pettenati J, Soler M, Camargo E L O, et al. Transcriptional regulation of the lignin biosynthetic pathway revisited: New players and insights. Adv Bot Res, 2012, 61: 173-218

32 Hiratsu K, Matsui K, Koyama T, et al. Dominant repression of target genes by chimeric repressors that include the EAR motif, a repression domain, in Arabidopsis. Plant J, 2003, 34: 733-739

33 Zhong R, Lee C, Ye Z H. Global analysis of direct targets of secondary wall NAC master switches in Arabidopsis. Mol Plant, 2010, 3: 1087-1103

34 Zhong R, Ye Z H. Transcriptional regulation of lignin biosynthesis. Plant Signal Behav, 2009, 4: 1028-1034

35 McCarthy R L, Zhong R, Fowler S, et al. The poplar MYB transcription factors, PtrMYB3 and PtrMYB20, are involved in the regulation of secondary wall biosynthesis. Plant Cell Physiol, 2010, 51: 1084-1090

36 Zhong R, Ye Z H. MYB46 and MYB83 bind to the SMRE sites and directly activate a suite of transcription factors and secondary wall biosynthetic genes. Plant Cell Physiol, 2012, 53: 368-380

37 Bonawitz N D, Chapple C. The genetics of lignin biosynthesis: Connecting genotype to phenotype. Annu Rev Genet, 2010, 44: 337-363

Zhao Q. Lignification: Flexibility, biosynthesis and regulation. Trends Plant Sci, 2016, 21: 713-721

39

Raes J, Rohde A, Christensen J H, et al. Genome-wide characterization of the lignification toolbox in Arabidopsis. Plant Physiol, 2003, 133: 1051-1071

40 Li E, Bhargava A, Qiang W, et al. The class II KNOX gene KNAT7 negatively regulates secondary wall formation in Arabidopsis and is functionally conserved in Populus. New Phytol, 2012, 194: 102-115

41 Chen H, Wang J P, Liu H, et al. Hierarchical transcription factor and chromatin binding network for wood formation in Populus trichocarpa. Plant Cell, 2019, 31: 602-626

42 Ohtani M, Nishikubo N, Xu B, et al. A NAC domain protein family contributing to the regulation of wood formation in poplar. Plant J, 2011, 67: 499-512

43 Zhong R, Lee C, Ye Z H. Functional characterization of poplar wood-associated NAC domain transcription factors. Plant Physiol, 2010, 152: $1044-1055$

44 Zhong R, McCarthy R L, Haghighat M, et al. The poplar MYB master switches bind to the SMRE site and activate the secondary wall biosynthetic program during wood formation. PLoS ONE, 2013, 8: e69219

45 Wang L, Lu W, Ran L, et al. R2R3-MYB transcription factor MYB6 promotes anthocyanin and proanthocyanidin biosynthesis but inhibits secondary cell wall formation in Populus tomentosa. Plant J, 2019, 99: 733-751 
46 Zhong R, McCarthy R L, Lee C, et al. Dissection of the transcriptional program regulating secondary wall biosynthesis during wood formation in poplar. Plant Physiol, 2011, 157: 1452-1468

47 Yang L, Hou Y, Zhao X, et al. Identification and characterization of a wood-associated NAC domain transcription factor PtoVNS11 from Populus tomentosa Carr. Trees, 2015, 29: 1091-1101

48 Lin Y C, Li W, Sun Y H, et al. SND1 transcription factor-directed quantitative functional hierarchical genetic regulatory network in wood formation in Populus trichocarpa. Plant Cell, 2013, 25: 4324-4341

49 Lin Y C J, Chen H, Li Q, et al. Reciprocal cross-regulation of VND and SND multigene TF families for wood formation in Populus trichocarpa. Proc Natl Acad Sci USA, 2017, 114: E9722-E9729

50 Wang H H, Tang R J, Liu H, et al. Chimeric repressor of PtSND2 severely affects wood formation in transgenic Populus. Tree Physiol, 2013, 33: $878-886$

51 Grant E H, Fujino T, Beers E P, et al. Characterization of NAC domain transcription factors implicated in control of vascular cell differentiation in Arabidopsis and Populus. Planta, 2010, 232: 337-352

52 Wang S, Li E, Porth I, et al. Regulation of secondary cell wall biosynthesis by poplar R2R3 MYB transcription factor PtrMYB152 in Arabidopsis. Sci Rep, 2014, 4: 5054

53 Li C, Wang X, Ran L, et al. PtoMYB92 is a transcriptional activator of the lignin biosynthetic pathway during secondary cell wall formation in Populus tomentosa. Plant Cell Physiol, 2015, 56: 2436-2446

54 Xie M, Muchero W, Bryan A C, et al. A 5-enolpyruvylshikimate 3-phosphate synthase functions as a transcriptional repressor in Populus. Plant Cell, 2018, 30: 1645-1660

55 Karpinska B, Karlsson M, Srivastava M, et al. MYB transcription factors are differentially expressed and regulated during secondary vascular tissue development in hybrid aspen. Plant Mol Biol, 2004, 56: 255-270

56 Wang Z G. Function characterization of six key MYB transcription factors involved in Populus wood formation (in Chinese). Dissertation for Master's Degree. Guangzhou: Jinan University, 2013 [王增光. 6个参与杨树木材形成的关键MYB转录因子的功能解析. 硕士学位论文. 广 州: 暨南大学, 2013]

57 Wilkins O, Nahal H, Foong J, et al. Expansion and diversification of the Populus R2R3-MYB family of transcription factors. Plant Physiol, 2009, 149: 981-993

58 Guo W, Zhao S T, Lu M Z. Analysis of MYB transcription factor involved in development of secondary vascular system in tobacco and Populus tomentosa (in Chinese). For Res, 2016, 29: 25-33 [郭伟, 赵树堂, 卢孟柱. 烟草和毛白杨次生维管发育相关MYB转录因子分析. 林业科学研 究, 2016, 29: 25-33]

59 Pyo H, Demura T, Fukuda H. Spatial and temporal tracing of vessel differentiation in young Arabidopsis seedlings by the expression of an immature tracheary element-specific promoter. Plant Cell Physiol, 2004, 45: 1529-1536

60 Escamez S, Tuominen H. Programmes of cell death and autolysis in tracheary elements: When a suicidal cell arranges its own corpse removal. J Exp Bot, 2014, 65: 1313-1321

61 Fukuda H. Tracheary element differentiation. Plant Cell, 1997, 9: 1147-1156

62 Aoyagi S, Sugiyama M, Fukuda H. BEN1 and ZEN1 cDNAs encoding S1-type DNases that are associated with programmed cell death in plants. FEBS Lett, 1998, 429: 134-138

63 Ito J, Fukuda H. ZEN1 is a key enzyme in the degradation of nuclear DNA during programmed cell death of tracheary elements. Plant Cell, 2002, 14: $3201-3211$

64 Pérez-Amador M A, Abler M L, De Rocher E J, et al. Identification of BFN1, a bifunctional nuclease induced during leaf and stem senescence in Arabidopsis. Plant Physiol, 2000, 122: 169-180

65 Farage-Barhom S, Burd S, Sonego L, et al. Expression analysis of the BFN1 nuclease gene promoter during senescence, abscission, and programmed cell death-related processes. J Exp Bot, 2008, 59: 3247-3258

66 Farage-Barhom S, Burd S, Sonego L, et al. Localization of the Arabidopsis senescence- and cell death-associated BFN1 nuclease: From the ER to fragmented nuclei. Mol Plant, 2011, 4: 1062-1073

67 Wang J G. Study the functon of PtCDD, a $\mathrm{Ca}^{2+}$ dependent nuclease, that is involved in secondary xylem development in Populus tomentosa (in Chinese). Dissertation for Doctoral Degree. Beijing: Chinese Academy of Forestry, 2015 [王俊刚. 毛白杨钙离子依赖的核酸酶PtCDD基因在 次生木质部发育中的功能研究. 博士学位论文. 北京: 中国林业科学研究院, 2015] 
68 Earnshaw W C, Martins L M, Kaufmann S H. Mammalian caspases: Structure, activation, substrates, and functions during apoptosis. Annu Rev Biochem, 1999, 68: 383-424

69 Beers E P, Freeman T B. Proteinase activity during tracheary element differentiation in Zinnia mesophyll cultures. Plant Physiol, 1997, 113: 873880

70 Ye Z H, Varner J E. Induction of cysteine and serine proteases during xylogenesis in Zinnia elegans. Plant Mol Biol, 1996, 30: $1233-1246$

71 Woffenden B J, Freeman T B, Beers E P. Proteasome inhibitors prevent tracheary element differentiation in Zinnia mesophyll cell cultures. Plant Physiol, 1998, 118: 419-430

72 Minami A, Fukuda H. Transient and specific expression of a cysteine endopeptidase associated with autolysis during differentiation of Zinnia mesophyll cells into tracheary elements. Plant Cell Physiol, 1995, 36: 1599-1606

73 Yamamoto R, Demura T, Fukuda H. Brassinosteroids induce entry into the final stage of tracheary element differentiation in cultured Zinnia cells. Plant Cell Physiol, 1997, 38: 980-983

74 Han J J, Lin W, Oda Y, et al. The proteasome is responsible for caspase-3-like activity during xylem development. Plant J, 2012, 72: 129-141

75 Funk V, Kositsup B, Zhao C, et al. The Arabidopsis xylem peptidase XCP1 is a tracheary element vacuolar protein that may be a papain ortholog. Plant Physiol, 2002, 128: 84-94

76 Avci U, Earl Petzold H, Ismail I O, et al. Cysteine proteases XCP1 and XCP2 aid micro-autolysis within the intact central vacuole during xylogenesis in Arabidopsis roots. Plant J, 2008, 56: 303-315

77 Bollhöner B, Zhang B, Stael S, et al. Post mortem function of AtMC9 in xylem vessel elements. New Phytol, 2013, 200: 498-510

78 Hara-Nishimura I, Hatsugai N. The role of vacuole in plant cell death. Cell Death Differ, 2011, 18: 1298-1304

79 Hatsugai N, Kuroyanagi M, Yamada K, et al. A plant vacuolar protease, VPE, mediates virus-induced hypersensitive cell death. Science, 2004, 305: $855-858$

80 Moreau C, Aksenov N, Lorenzo M G, et al. A genomic approach to investigate developmental cell death in woody tissues of Populus trees. Genome Biol, 2005, 6: R34

81 Courtois-Moreau C L, Pesquet E, Sjödin A, et al. A unique program for cell death in xylem fibers of Populus stem. Plant J, 2009, 58: 260-274

82 Cheng Z, Zhang J, Yin B, et al. $\gamma \mathrm{VPE}$ plays an important role in programmed cell death for xylem fiber cells by activating protease CEP1 maturation in Arabidopsis thaliana. Int J Biol Macromol, 2019, 137: 703-711

83 Zhang D, Liu D, Lv X, et al. The cysteine protease CEP1, a key executor involved in tapetal programmed cell death, regulates pollen development in Arabidopsis. Plant Cell, 2014, 26: 2939-2961

84 Han J, Li H, Yin B, et al. The papain-like cysteine protease CEP1 is involved in programmed cell death and secondary wall thickening during xylem development in Arabidopsis. J Exp Bot, 2019, 70: 205-215 


\title{
The molecular mechanism in secondary wall deposition and programmed cell death of wood formation
}

\author{
LI Hui ${ }^{1,2,3}$, GUO XiaoRui ${ }^{1}$, LIU YaLin ${ }^{1} \&$ LU Hai ${ }^{1,2,3}$ \\ 1 College of Biological Sciences and Technology, Beijing Forestry University, Beijing 100083, China; \\ 2 National Engineering Laboratory for Tree Breeding, Beijing Forestry University, Beijing 100083, China; \\ 3 Beijing Advanced Innovation Center for Tree Breeding by Molecular Design, Beijing Forestry University, Beijing 100083, China
}

Wood formation occurs through continuous steps involving cambium cell proliferation, xylem cell specification and expansion, secondary wall deposition and programmed cell death (PCD). Recently, many key transcription factors/repressors and enzymes involved in secondary wall formation and PCD processes have been identified based on genetics and omics analysis in model plants Arabidopsis and poplar. All these hierarchical transcription factors build the transcriptional regulatory network of secondary wall deposition. These transcription factors not only play important role in regulating the biosynthesis of secondary wall components including lignin, cellulose and xylan, but also activate downstream hydrolytic enzymes and trigger PCD process. To analyze the biological function and regulatory network of these genes, it will provide the theoretical foundation for elucidating the molecular mechanism of wood formation. In this paper, we review the advances of transcriptional regulatory network in secondary wall deposition and enzymatic mechanism in PCD of wood formation.

wood, secondary wall, transcription factors, PCD, protease

doi: $10.1360 /$ SSV-2019-0133 